\title{
RESEARCH
}

Open Access

\section{Plasma lipidome abnormalities in people with HIV initiating antiretroviral therapy}

\author{
Emily R. Bowman ${ }^{1 *}$ (D), Manjusha Kulkarni ${ }^{1}$, Janelle Gabriel ${ }^{1}$, Xiaokui Mo ${ }^{1}$, Brett Klamer ${ }^{1}$, Martha Belury ${ }^{1}$, \\ Jordan E. Lake², David Zidar', Scott F. Sieg ${ }^{3}$, Nehal N. Mehta ${ }^{4}$, Martin P. Playford', Daniel R. Kuritzkes ${ }^{5}$, \\ Adriana Andrade ${ }^{6}$, Elizabeth Koss Schmidt ${ }^{1}$, Christopher Taylor ${ }^{1}$, Edgar T. Overton? ${ }^{7}$, Amanda L. Willig ${ }^{7}$, \\ Michael M. Lederman ${ }^{3}$, Nicholas T. Funderburg ${ }^{1}$ and the AIDS Clinical Trials Group A5248/A5249s Team
}

\begin{abstract}
Background: Dyslipidemia often accompanies human immunodeficiency virus (HIV) infection and antiretroviral therapy (ART). Lipid abnormalities likely contribute to increased cardiometabolic disease among people with HIV $(\mathrm{PWH})$. Here, we expand our previous findings on changes in the lipidome following ART initiation, and associations among lipid species, including ceramides (CER), diacylglycerols (DAG), and triacylglycerols (TAG), with immune activation.

Methods: Concentrations and fatty acid composition of plasma lipids ( 1300 species) were measured by differential mobility spectroscopy in samples from 35 treatment-naïv PWH pre- and post-initiation of ART (raltegravir (RAL)/ tenofovir disoproxil fumarate (TDF)/emtricitabine (FTC)); lipidomes were compared to those found in demographically similar HIV-uninfected individuals $(n=13)$.

Results: Compared to people without HIV, 37.1\% of all lipid species measured were altered in PWH at baseline, and $31.8 \%$ of lipid species were altered following 48 weeks of ART. Concentrations of lipid classes were also altered in PWH; diacylglycerols (DAGs) and triacylglycerols (TAGs) were increased at baseline, and DAGs remained increased after 48 weeks of ART. Lipids previously linked to cardiovascular disease (CVD) and diabetes were enriched in PWH pre- and post ART, and were related to immune activation and insulin resistance scores. Polyunsaturated fatty acid (PUFA)containing lipids were lower in PWH compared to levels in controls, and were inversely related to levels of inflammatory biomarkers.
\end{abstract}

Conclusions: HIV infection and ART initiation both induce cardiometabolic changes to the composition of the plasma lipidome. These alterations are associated with inflammatory biomarkers, and may directly contribute to elevated CVD risk and diabetes.

Trial registration: This study is registered with Clinicaltrials.gov (NCT00660972). Registered April 16, 2008.

Keywords: Lipidome, HIV, Antiretroviral therapy, Inflammation, Cardiovascular disease

\footnotetext{
* Correspondence: Emily.Bowman@osumc.edu

${ }^{1}$ The Ohio State University, 333 W. 10th Ave. 5198 Graves Hall, Columbus, $\mathrm{OH}$ 43210, USA

Full list of author information is available at the end of the article
}

(c) The Author(s). 2020 Open Access This article is licensed under a Creative Commons Attribution 4.0 International License, which permits use, sharing, adaptation, distribution and reproduction in any medium or format, as long as you give appropriate credit to the original author(s) and the source, provide a link to the Creative Commons licence, and indicate if changes were made. The images or other third party material in this article are included in the article's Creative Commons licence, unless indicated otherwise in a credit line to the material. If material is not included in the article's Creative Commons licence and your intended use is not permitted by statutory regulation or exceeds the permitted use, you will need to obtain permission directly from the copyright holder. To view a copy of this licence, visit http://creativecommons.org/licenses/by/4.0/ 


\section{Background}

HIV infection and antiretroviral therapy (ART) have been associated with an increased risk for cardiovascular disease (CVD) [1]. Dyslipidemia is a common manifestation of HIV infection, with reduced levels of high-density lipoprotein (HDL) cholesterol, and elevated low-density lipoprotein (LDL) cholesterol, total cholesterol (TC), and triglycerides (TG) frequently observed in ART-treated PWH. This lipid profile has been linked to progressive atherosclerosis in the general population; however conventional lipid measurements may not adequately predict cardiometabolic risk among people with HIV (PWH). Advances in mass-spectrometry-based lipid quantitation now allow for more detailed and extensive measurements of the lipidome and may provide a more accurate assessment of cardiometabolic risk in PWH [2].

Perturbations in lipid profiles in PWH are complicated further by excess immune activation that characterizes chronic HIV infection; inflammatory processes alter lipid metabolism, and certain lipid species also exacerbate inflammation. Thus, immune activation may be both a cause and a consequence of lipid abnormalities in HIV infection.

Here, in a supplemental study of the AIDS Clinical Trials Group (ACTG) A5248 trial [3], we profiled plasma concentrations and fatty acid composition of approximately 1300 different lipid species across 14 lipid classes in longitudinal samples from treatment-naïve PWH initiating a raltegravir (RAL)-based ART regimen, and following 48 weeks of ART. These results were compared to cross-sectional samples obtained from age and sex matched individuals without HIV. We report alterations in concentrations and fatty acid composition of the complete measured lipidome, including ceramides (CER), diacylglycerols (DAG), and triacylglycerols (TAG) that have been linked to CVD and diabetes in other studies [4, 5]. We also examine relationships among these lipids and systemic markers of immune activation.

\section{Materials and methods Study design}

This is an exploratory substudy of the ACTG A5248 clinical trial aimed at characterizing virologic and immunologic changes [3], in ART-naïve participants $(N=$ 39) who were treated for 48 weeks with RAL ( $400 \mathrm{mg}$ twice daily) and emtricitabine/tenofovir disoproxil fumarate $(200 \mathrm{mg} / 300 \mathrm{mg}$ once daily). All participants with HIV achieved virologic suppression by week 48 . Samples from age- and sex-matched HIV-uninfected participants $(N=13)$ were also analyzed. This study was approved by the institutional review boards at all participating sites [3], and is registered with Clinicaltrials.gov (NCT00660972).
Blood samples from A5248 study participants and participants without HIV were collected in EDTAcontaining tubes at baseline and at week 48 after ART initiation. Following centrifugation, plasma was removed and stored at $-80 \mathrm{C}$ until analysis.

\section{Lipid metabolite measurement}

The concentration $(\mu \mathrm{M})$ and fatty acid composition (mol\%) of approximately 1300 lipid metabolites from 14 different lipid classes were measured by Metabolon (True Mass Complex Lipid Panel, Metabolon, Research Triangle Park, NC). The lipid classes measured include the following: Cholesterol ester (CE), ceramide (CER), diacylglycerol (DAG), dihydroceramide (DCER), free fatty acid (FFA), hexosylceramide (HCER), lactosylceramide (LCER), lysophosphatidylcholine (LPC), lysophosphatidylethanolamine (LPE), phosphatidylcholine (PC), phosphatidylethanolamine (PE), phosphatidylinositol (PI), sphingomyelin (SM), triacylglycerol (TAG). Plasma lipids were extracted in methanol:dichloromethane, and concentrated under nitrogen. The extracts were analyzed by mass spectroscopy using a Shimadzu LC with nanoPEEk tubing and the Sciex SelexIon-5500QTRAP. Lipid class concentrations $(\mu \mathrm{M})$ were calculated from the sum of all molecular species within a class, and fatty acid composition (mol\%) measurements were determined by calculating the proportion of each class comprised by individual fatty acids. NMR spectroscopy was used for lipid particle phenotyping. The lipoprotein insulin resistance (LPIR) score was calculated by Liposcience Labcorp (Burlington, NC), using a weighted algorithm based on VLDL, LDL, and HDL particle sizes. The 50th percentile has an LPIR of 45, with 0 indicating insulin sensitivity and 100 indicating insulin resistance [6].

\section{Plasma biomarker assays}

Levels of soluble CD14 (sCD14), soluble tumor necrosis factor receptor 1 (sTNF-R1), intracellular adhesion molecule (ICAM)-1, vascular cell adhesion molecule (VCAM)-1, and CX3CL1 (Fractalkine) were measured using Quantikine ELISA kits (R\&D Systems). Plasma levels of lipopolysaccharide (LPS) were quantified using the Limulus Amebocyte Lysate (LAL) assay (QCL-1000, Lonza, Walkersville, MD).

\section{Statistical analysis}

The differences in concentrations and composition of the lipidomes among PWH and participants without HIV were analyzed by 2 -sample $t$ tests, and the differences between levels at baseline and after 48 weeks of ART were analyzed by paired $t$ tests. The associations among lipid levels and biomarkers of inflammation 
were calculated using Spearman correlation methods. The associations among demographics, sex and race, and HIV status were tested by Fisher's Exact tests. Data analysis was performed in SAS 9.4 (SAS, INC; Cary, NC).

\section{Results}

Plasma samples were collected from 35 PWH (A5248 study participants, untreated and after 48 weeks of suppressive ART), and from 13 participants without HIV. Demographic information is shown in Table 1. Among PWH, levels of total cholesterol (TC), HDL, and LDL increased from baseline to week 48. LPIR scores, predictive of type 2 diabetes risk [6], also tended to be increased in PWH at baseline and week 48 compared to participants without HIV.
The concentration and fatty acid composition of lipids is altered by HIV infection and by ART

We report that the overall concentrations of triacylglycerols (TAG) and diacylglycerols (DAG) in PWH at baseline $(1531 \mu \mathrm{M}$ and $3 \mu \mathrm{M})$ are greater than those found in individuals without $\mathrm{HIV}(1036 \mu \mathrm{M}, p=0.05 ; 1.7 \mu \mathrm{M}, p=$ 0.02 , respectively); DAG levels remained significantly higher among PWH than levels in people without HIV after 48 weeks of ART ( $2.8 \mu \mathrm{M}, p=0.02)$ (Fig. 1).

We measured significantly lower concentrations of cholesterol esters (CE) $(3163 \mu \mathrm{M}$ vs $3978 \mu \mathrm{M})$, phosphatidylcholine (PC) $(1268 \mu \mathrm{M}$ vs $1634 \mu \mathrm{M})$, sphingomyelin (SM) $(343 \mu \mathrm{M}$ vs $426 \mu \mathrm{M})$, and lactosylceramides (LCER) $(3 \mu \mathrm{M}$ vs $3.6 \mu \mathrm{M})(p<0.05$ for all $)$ in PWH at baseline compared to concentrations in the individuals without HIV. After week 48 of ART, levels of PC $(1350 \mu \mathrm{M})$, SM $(371 \mu \mathrm{M})$, and LCER $(3 \mu \mathrm{M})$ remained significantly

Table 1 Study participant characteristics

\begin{tabular}{|c|c|c|c|c|}
\hline & HIV- $(n=13)$ & PWH, Baseline $(n=35)$ & PWH, Week $48(n=35)$ & $P$-Values \\
\hline Age & $43(23-58)$ & $43(23-58)$ & $44(24-59)$ & $P=0.98$ \\
\hline HIV Viral Load (copies/mL) & NA & $37,153(6637-619,797)$ & $48(48-48)$ & \\
\hline CD4+ T cell (cells/mL) & NR & $259(1-599)$ & $489(87-1026)$ & \\
\hline \multirow[t]{2}{*}{ Sex (\%) } & Male $=10(77 \%)$ & Male = $32(91 \%)$ & & $P=0.32$ \\
\hline & Female = $3(23 \%)$ & Female = $3(9 \%)$ & & \\
\hline \multirow[t]{3}{*}{ Ethnicity (\%) } & White $=9(69 \%)$ & White = $18(52 \%)$ & & $P=0.35$ \\
\hline & Not White $=4(31 \%)$ & Not White = $12(34 \%)$ & & \\
\hline & & Hispanic = $5(14 \%)$ & & \\
\hline \multirow[t]{3}{*}{ Total Cholesterol (mg/dL) } & $185(93-266)$ & $125(87-208)$ & $142(93-226)$ & $P<0.001, \mathrm{HIV}-\vee \mathrm{BL}$ \\
\hline & & & & $P=0.005, \mathrm{HIV}-\mathrm{V}$ Wk48 \\
\hline & & & & $P=0.004, B L \vee W k 48$ \\
\hline \multirow[t]{3}{*}{$\mathrm{LDL}(\mathrm{mg} / \mathrm{dL})$} & $100(34-176)$ & $73(51-154)$ & $84(45-164)$ & $P=0.02, \mathrm{HIV}-\vee \mathrm{BL}$ \\
\hline & & & & $P=0.17, \mathrm{HIV}-\vee \mathrm{Wk} 48$ \\
\hline & & & & $P=0.02, B L \vee W k 48$ \\
\hline \multirow[t]{3}{*}{$\mathrm{HDL}(\mathrm{mg} / \mathrm{dL})$} & $57(41-103)$ & $35(0-56)$ & $41(17-71)$ & $P<0.001, \mathrm{HIV}-\vee \mathrm{BL}$ \\
\hline & & & & $P<0.001$, HIV- v Wk48 \\
\hline & & & & $P=0.004, B L \vee W k 48$ \\
\hline \multirow[t]{3}{*}{ Triglycerides (mg/dL) } & $89(62-205)$ & $72(36-162)$ & $87(30-200)$ & $P=0.48, \mathrm{HIV}-\vee \mathrm{BL}$ \\
\hline & & & & $P=0.95, \mathrm{HIV}-\vee \mathrm{Wk} 48$ \\
\hline & & & & $P=0.34, B L \vee W k 48$ \\
\hline \multirow[t]{3}{*}{ Lipoprotein Insulin Resistance (LPIR) score } & $42(1-70)$ & $52(10-77)$ & $53(10-86)$ & $P=0.06, \mathrm{HIV}-\vee \mathrm{BL}$ \\
\hline & & & & $P=0.2, \mathrm{HIV}-\vee \mathrm{Wk} 48$ \\
\hline & & & & $P=0.42, B L \vee W k 48$ \\
\hline Adiponectin (ng/mL) & NR & $4281(558-14,652)$ & $3600(446-8862)$ & $P=0.1$ \\
\hline Leptin $(p g / m L)$ & NR & $2412(325-81,416)$ & $5856(486-79,755)$ & $P=0.06$ \\
\hline $\mathrm{BMI} \geq 25$ (\%) & NR & 50 & NR & \\
\hline
\end{tabular}

Demographics and clinical characteristics of HIV-uninfected individuals and A5248 study participants, at baseline and after 48 weeks of ART. Data are displayed as medians and ranges

NR Not Recorded 


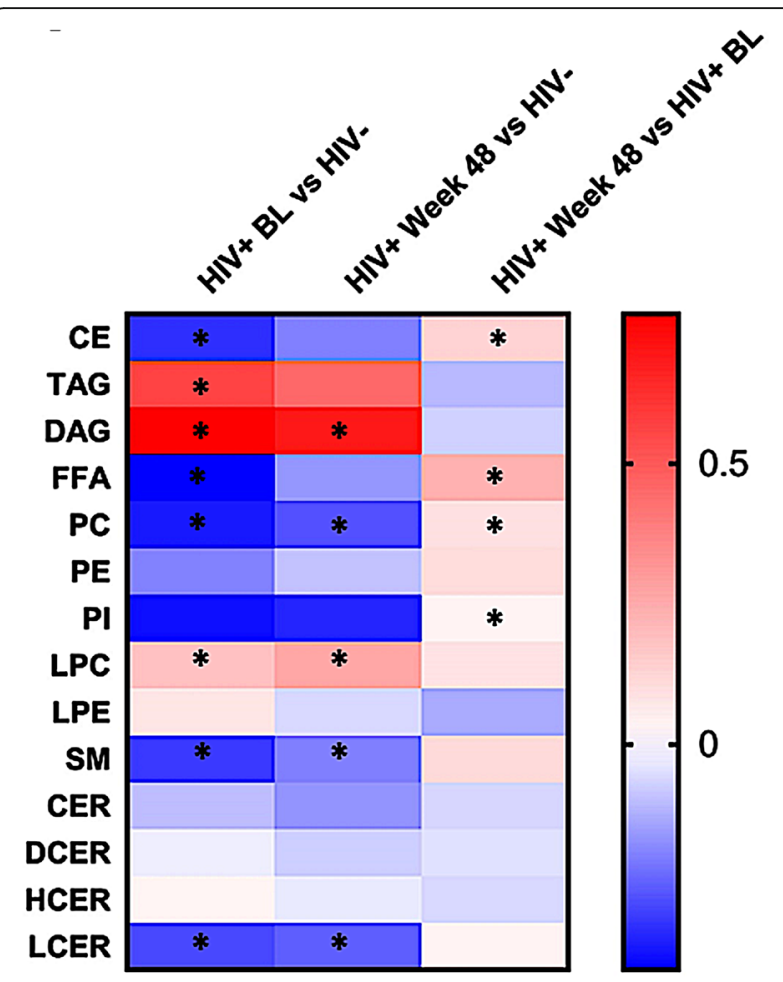

Fig. 1 The concentrations of lipid classes are altered by HIV infection and by ART. Lipid class concentrations $(\mu \mathrm{M})$ were calculated from the sum of all molecular species within a selected class. Statistical significance between study groups with and without HIV was determined using 2-sample unpaired t tests, and among samples from PWH at baseline and after 48 weeks of ART, paired t testing was used. Heatmap data are represented as Fold Log2. $\left({ }^{*}<0.05\right)$

reduced among $\mathrm{PWH}$ compared to levels in persons without HIV ( $p<0.05$ for all). However, ART treatment resulted in increased levels of CE $(3464 \mu \mathrm{M}$ vs $3163 \mu \mathrm{M}$, $p=0.05)$, free fatty acids (FFA) $(352 \mu \mathrm{M}$ vs $299 \mu \mathrm{M}, p=$ $0.004)$, PC $(1350 \mu \mathrm{M}$ vs $1268 \mu \mathrm{M}, p=0.03)$, and phosphatidylinositol (PI) $(17 \mu \mathrm{M}$ vs $16.5 \mu \mathrm{M}, p=0.03)$, relative to baseline levels in the PWH population (Fig. 1).

We detected broad alterations in concentrations of individual lipid species among PWH (baseline to week 48) and compared to individuals without HIV (for complete list of lipid species concentrations, see Additional Table 1). Compared to the people without HIV, $32.2 \%$ of all lipid species were significantly increased and $4.9 \%$ were decreased in PWH at baseline, and 29.6\% of lipids were increased and $2.2 \%$ were decreased in PWH following 48 weeks of ART. Further, after 48 weeks of ART we measured an increase in $7.6 \%$ of all lipids and a decrease in $4.7 \%$ of lipids compared to baseline levels in PWH. For a comprehensive list of the concentrations and proportional representation ( $\mathrm{mol} \%$ ) of the numerous lipid species that are significantly altered among PWH, see Additional Tables 1 and 2 .
Lipids associated with cardiovascular disease risk are increased in PWH, and are associated with inflammatory biomarkers

Multiple individual TAG species are predictive of CVD risk and diabetes in the general population $[4,5,7,8]$. Concentrations of these TAGs also tended to be increased in PWH at baseline compared to levels in participants without HIV, with TAG(51:2), TAG(54:2), TAG(54:3), TAG(56:1), and TAG(56:5) differences reaching statistical significance $(p<0.05)$ (Additional Table 3). After 48 weeks of ART, the concentrations of TAG(54:2), TAG(54:3), and TAG(56:5) were decreased relative to baseline $(p<0.05)$.

The fatty acid composition of TAG species has been linked to CVD and diabetes, as TAGs containing SaFAs (palmitic acid (16:0), stearic acid (18:0)) and oleic acid (18:1) were associated with cardiometabolic risk [4, 5, 8]. We analyzed fatty acid composition of TAGs, and observed increased concentrations of risk-associated TAGs composed of SaFAs and oleic acid (for a complete list of lipid concentrations, see Additional Table 1). We also detected correlations among TAG species composed of these fatty acids and inflammatory markers and traditional lipid measurements (Fig. 2). Overall, TAGs were directly related to levels of sTNFR1, sCD14, LPS, low density lipoprotein (LDL), very low density lipoprotein (VLDL), and lipoprotein insulin resistance (LPIR) score, a metabolomic biomarker associated with diabetes risk; TAGs were inversely associated with HDL levels $(p<$ 0.05 for all). After 48 weeks of ART, many of the associations among TAG species and sTNFR1, sCD14, LPS, LDL, and HDL were no longer significant; however, the associations with LPIR and VLDL remained significant.

Ceramide (CER) levels, and CER (16:0) in particular, are also predictive of CVD and diabetes in the general population and levels of ceramides were associated with ART use and carotid atherosclerosis in PWH [9]. Here, the proportional representation of CER (16:0) was increased significantly in PWH at both baseline $(7.6 \mathrm{~mol} \%$, $p=0.013)$ and after 48 weeks of ART ( $7.5 \mathrm{~mol} \%, p=$ $0.015)$ compared to levels in individuals without HIV (6.1 mol\%) (for a complete list of lipid composition, see Additional Table 2). We measured direct associations among levels of CER (16:0) after 48 weeks of ART and the inflammatory biomarkers, ICAM-1 $(\mathrm{r}=0.48, p=$ $0.005)$ and sTNFR1 $(r=0.53, p=0.04)$ (Fig. 3a). Viral suppression with ART reduces levels of immune activation in PWH, however often not to levels observed in people without HIV. ICAM-1 and sTNFR1 concentrations were reduced in PWH following initiation of ART, but remain correlated with CVD-associated lipid levels (Additional Table 4). The proportional representation of PC (18:0/20:3), a lipid previously found to be enriched in atherosclerotic plaques, was also increased in $\mathrm{PWH}$ at 

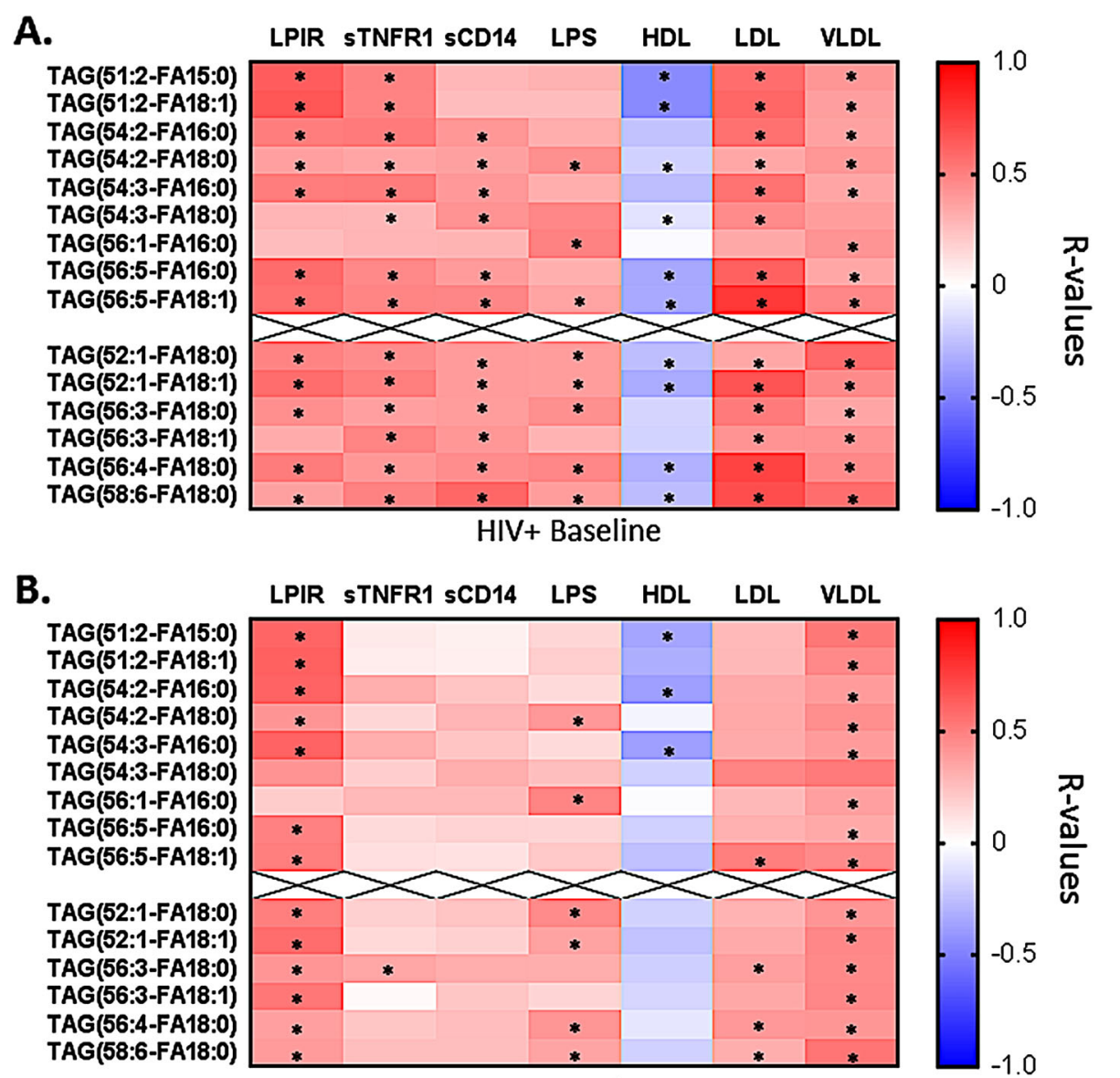

HIV+ Week 48

Fig. 2 TAG species composed of saturated fatty acids are associated with inflammatory biomarkers and clinical indices. Associations among concentrations $(\mu \mathrm{M})$ of TAGs and inflammatory and clinical markers from samples from $\mathbf{a}$ untreated HIV+ individuals and $\mathbf{b}$ following 48 weeks of ART are reported. TAG species above the hashmark have been previously associated with CVD, and TAGs below the hashmark are species that have not been linked to CVD in the general population, but have similar relationships to inflammatory biomarkers and traditional lipid measurements. Spearman correlations are reported for all associations. $\left.{ }^{*} p<0.05\right)$

baseline $(1.51 \mathrm{~mol} \%, p=0.006)$ and after 48 weeks of ART $(1.45 \mathrm{~mol} \%, p=0.054)$ compared to levels in individuals without HIV (1.14 mol\%) (see Additional Table 2). There was a direct relationship between $P C$ (18:0/20: $3)$ and oxidized LDL (OxLDL) levels $(r=0.53, p=0.002)$ (Fig. 3b).

PUFA-containing cholesterol esters (CEs), such as CE (18:3) and CE (20:5), have been linked previously to plaque stability and reduced CVD risk [2]. Compared to individuals without HIV, concentrations of CE (18:3) and CE (20:5) are reduced in PWH at baseline $(71.4 \mu \mathrm{M}$ v. $53.7 \mu \mathrm{M}, p=0.04 ; 25.6$ v. $17.2, p=0.03$, respectively) (for a complete list of lipid species concentrations, see Additional Table 1). Following 48 weeks of ART, concentration of CE (20:5) significantly increased to levels comparable to people without HIV $(17.2 \mu \mathrm{M}$ v. $24.3 \mu \mathrm{M}$, $p=0.03)$. Further, levels of CE (18:3) were inversely related to biomarkers of endothelial cell activation, ICAM$1(r=-0.46, p=0.01)$ and VCAM-1 $(r=-0.40, p=0.03)$, and CE (20:5) was inversely related to CX3CL1 (fractalkine) $(r=-0.40, p=0.03)$ (Fig. 3b).

\section{Discussion}

Both HIV infection and ART treatment alter lipid profiles [1], however, many published studies focus primarily on conventional lipid panels (TC, HDL, LDL), which may not adequately assess cardiometabolic risk in HIV infection [2]. In this analysis, we detail changes in the concentrations and proportional representation of approximately 1300 lipid species in treatment naïve PWH at baseline and after 48 weeks of ART. Compared to individuals without HIV, PWH had significant increases in the overall concentration of TAGs, DAGs, and LPCs, which are lipid classes previously implicated in CVD [7] and diabetes $[4,5]$. Despite suppressive ART treatment, concentrations of these lipid classes remained elevated. Furthermore, ART treatment resulted in significant increases from baseline in total concentrations of CEs, 

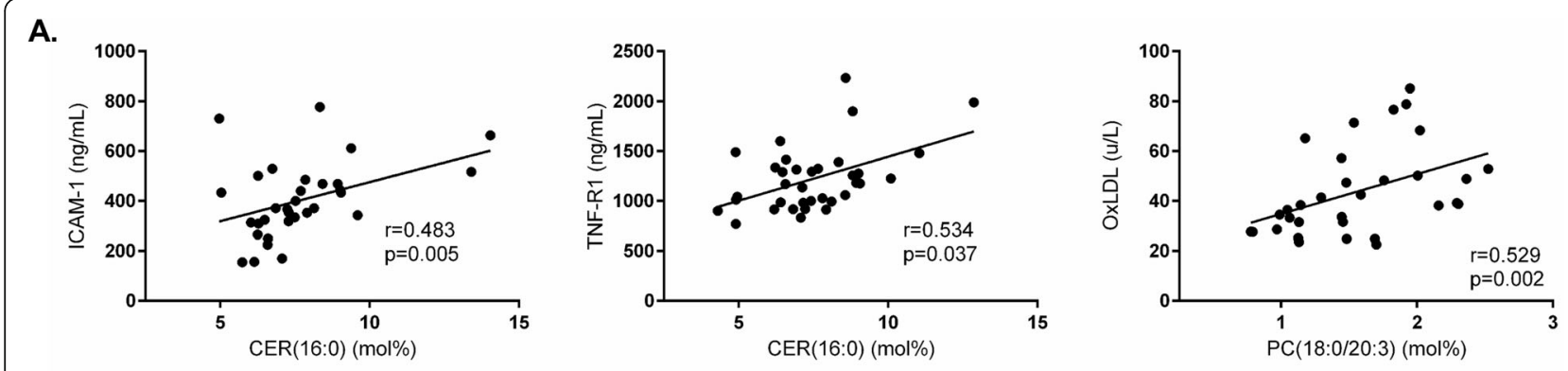

B.
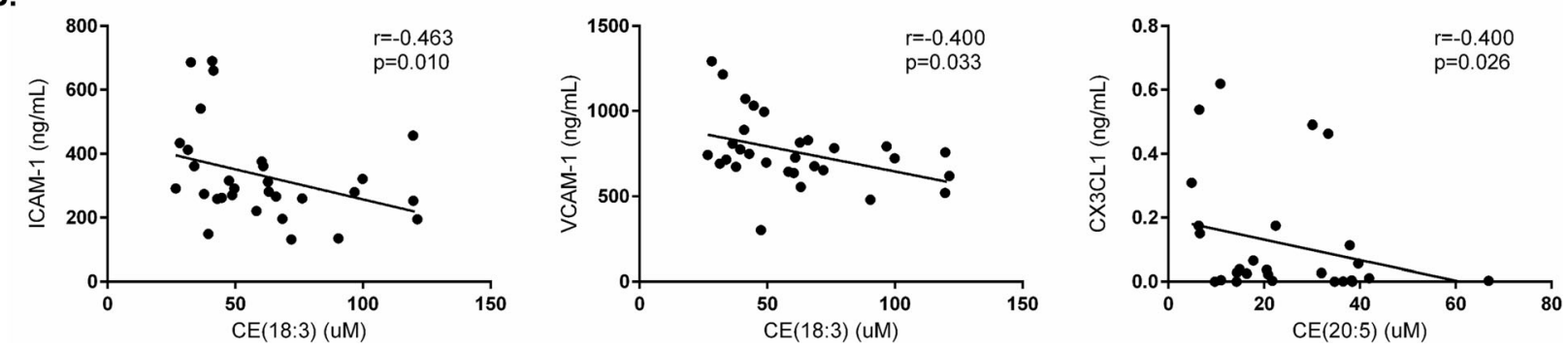

Fig. 3 Lipid species associated with CVD risk correlate with soluble biomarkers of inflammation Plasma samples were thawed and levels of soluble inflammatory biomarkers (ICAM-1, VCAM-1, sTNF-R1, CX3CL1, OxLDL) were measured by ELISA. a Spearman correlations demonstrate direct relationships among inflammatory marker levels and lipid species positively associated with CVD risk. $\mathbf{b}$ Levels of lipid species previously shown to be negatively associated with CVD risk were also negatively associated with inflammatory markers in PWH after initiation of ART

FFAs, PCs, and PIs. ART drugs have varying adverse effects on lipid metabolism, and the use of which may also contribute to increased risk of cardiometabolic diseases, including diabetes, atherosclerosis and CVD [2].

In people without HIV, total TAG levels have been linked to insulin resistance and diabetes, and are implicated as an independent risk factor for CVD [5]. Several recent analyses, however, have identified specific TAG species as being more strongly predictive of CVD than total TAG levels $[5,7,8]$. In our study, many of these CVD-associated TAG species, including TAG (51:2), TAG (54:2), TAG (54:3), TAG (56:1), TAG (56:5), were also elevated in PWH at baseline, although after 48 weeks of ART some of these concentrations decreased (Additional Table 3). These TAG species were also directly related to the inflammatory biomarkers sTNF-R1 and $\mathrm{sCD} 14$, both of which have been linked to increased morbidity and mortality in HIV infection (Fig. 2), however, ART treatment subsequently reduced levels of sTNF-R1 $(1522 \mathrm{pg} / \mathrm{mL}$ to $1222 \mathrm{pg} / \mathrm{mL})$ and $\mathrm{sCD} 14$ (2051 ng/mL to $1661 \mathrm{ng} / \mathrm{mL}$ ) in PWH, and associations with CVD-associated TAGs were no longer significant.

Multiple TAGs were also directly associated with LPIR score, an index associated with insulin resistance and diabetes risk. The early detectable stages of insulin resistance include alterations in lipid levels and lipoprotein metabolism [6]. LPIR testing, which takes into account VLDL, LDL, and HDL levels, has been used to improve diabetes prevention efforts by identifying potential insulin-resistant patients with normal blood glucose levels and $\beta$-cell function [6]. Following 48 weeks of ART, TAG levels declined and many significant correlations among inflammatory biomarkers were lost, but LPIR score, which did not improve with ART, remained directly associated with CVD-associated TAGs.

The fatty acid composition of TAGs is important, as TAGs containing palmitic acid (16:0), stearic acid (18:0), and oleic acid (18:1) have been more strongly associated with CVD than are TAGs containing other fatty acids [8]. Similarly, in our study, the strongest relationships to inflammatory biomarkers and clinical indices were among the TAGs composed of SaFAs and oleic acid (18:1). Chronic inflammation likely underlies the progression of many diseases, including HIV, and SaFAs may be one driver of persistent inflammation. Alterations in SaFA, MUFA, and PUFA concentrations and fatty acid composition of total, free (non-esterified), and LPC lipid species have similarly been reported in PWH initiating a Raltegravirbased ART regimen [10].

In addition to the previously reported CVD-associated TAGs, we also identified additional TAG species (TAG(52:1), TAG(56:3), TAG(56:4), TAG(58:6)) that were elevated in PWH and were similarly related to markers of inflammation and clinical indices (Additional Table 3, Fig. 2). These TAG species may prove to be 
important for HIV-related disease progression in future studies.

Other lipid classes, including CER, may be predictive of cardiometabolic complications in PWH. In our study, total CER levels were not significantly different among people with and without HIV, however, we did detect differences in the amount of certain CER species, including CER (16:0) and CER (24:0), which have been linked to CVD and all-cause mortality [9]. Furthermore, proportional amounts of CER (16:0) measured in PWH at baseline were directly associated with plasma biomarkers of inflammation, ICAM-1 and sTNF-R1. In PWH, we also measured proportional increases of the lipid species PC (18:0/20:3), previously shown to be enriched in atherosclerotic plaques in the general population.

There are limitations to this report. We were unable to account for dietary intake in our study participants, and diet likely has important effects on the overall lipidome. We do not, however, anticipate considerable dietary changes among study participants within 48 weeks. Nevertheless, future studies examining lipids and cardiometabolic health in PWH would be improved by the addition of dietary assessments and body composition information. In PWH at baseline, we did not observe significant associations among CVD-associated TAGs and body mass index (BMI), nor did we detect significant differences in TAG levels when grouped based on BMI $(<25$ vs $>25)$ (Data not shown). All of the PWH were also initiating an identical ART regimen; ART drugs can have varying effects on lipid metabolism $[1,10]$, and the lipid profiles that we observed in this study may differ in individuals taking a different regimen. Additionally, lipid levels and composition may be affected differently by long term ART usage, and here we only report lipid data after 48 weeks of ART. With a small proportion of female participants, our study was not powered to compare lipidome alterations among male and female participants, and future larger-scale studies should aim to elucidate potential links among sex differences, CVD risk, and lipidome alterations in people with HIV. The data generated here are exploratory, as the parent study was not powered for lipidomics analyses and we could not correct for multiple comparisons.

Our findings indicate that both HIV infection and ART induce broad lipidomic changes that may result in lipid profiles with enhanced cardiometabolic risk. Importantly, although ART often leads to controlled viral replication and reduces inflammation, our data suggest ART administration does not necessarily lead to the reversal of HIV-induced pro-atherosclerotic lipid profiles. Additional approaches beyond virus control through current ART regimens are needed to counter dyslipidemia and attenuate CVD risk. Detailed lipidomics analyses will likely prove to be an effective tool in predicting and evaluating CVD outcomes in PWH. Further work is needed to elaborate on drivers of dyslipidemia, and the interactions among the lipidome, chronic inflammation, and comorbidities in PWH.

\section{Supplementary Information}

The online version contains supplementary material available at https://doi. org/10.1186/s41231-020-00079-6.

Additional file 1: Table 1. Lipid species concentrations. Plasma samples were thawed and the concentrations $(\mu \mathrm{M})$ of the most abundant lipid metabolites ( 1300) from 14 different lipid classes were measured by Complex Lipid Panel (Metabolon). Mean levels are reported. Statistical significance between study groups with and without HIV was determined using 2-sample unpaired t tests, and among samples from PWH at baseline and after 48 weeks of ART, paired t testing was used. $P<$ 0.05 was used for significance cutoff, and significant values are shown in bold.

Additional file 2: Table 2. Lipid species composition. Plasma samples were thawed and the proportional representation (mol\%) among the most abundant lipid metabolites ( 1300) from 14 different lipid classes were measured by Complex Lipid Panel (Metabolon). Mean levels are reported. Statistical significance between study groups with and without HIV was determined using 2-sample unpaired t tests, and among samples from $\mathrm{PWH}$ at baseline and after 48 weeks of ART, paired t testing was used. $P<0.05$ was used for significance cutoff, and significant values are shown in bold.

Additional file 3: Table 3. Concentrations of TAGs previously linked to CVD are altered in PWH. Plasma samples were thawed and concentrations $(\mu \mathrm{M})$ of lipid molecules were measured by Complex Lipid Panel (Metabolon). Concentrations of TAGs previously shown to be associated with CVD risk were altered in $\mathrm{PWH}$.

Additional file 4: Table 4. Inflammatory biomarker levels following initiation of ART. Plasma samples from PWH were thawed and levels of soluble inflammatory biomarkers (ICAM-1, VCAM-1, sTNF-R1, CX3CL1, OxLDL) were measured by ELISA. Mean concentrations are reported at baseline and following 48 weeks of ART. Statistical significance was determined using paired t tests.

\section{Abbreviations}

ART: Antiretroviral therapy; PWH: People with HIV; CVD: Cardiovascular disease; TDF: Tenofovir disoproxil fumarate; FTC: Emtricitabine;

RAL: Raltegravir; CER: Ceramide; DAG: Diacylglyceride; TAG: Triacylglyceride; PUFA: Polyunsaturated fatty acid; MUFA: Monounsaturated fatty acid; SaFA: Saturated fatty acid; HDL: High-density lipoprotein; LDL: Low-density lipoprotein; VLDL: Very low-density lipoprotein; TG: Triglyceride; LPIR: Lipoprotein insulin resistance

\section{Acknowledgments}

Not applicable.

\section{Authors' contributions}

All authors contributed to experimental design, data analysis, and writing of the manuscript. The author(s) read and approved the final manuscript.

\section{Funding}

The project described was supported by grants from the National Institutes of Health grant numbers R00HL108743, R56HL126563, R01HL134544 (to NF), Al-07164, Al-67039, the Fasenmyer Foundation (Cleveland, OH), the Center for AIDS Research at Case Western Reserve University Al-36219, K23 Al110532 (to JEL), and an NIH Intramural Grant from the NHLBI to NNM (HL00619301). This work was also supported by Award Numbers UM1 Al068634, UM1 Al068636, and UM1 Al106701 from the National Institute of Allergy and Infectious Diseases of the National Institutes of Health. This content is solely the responsibility of the authors and does not necessarily represent the official views of the National Institutes of Health. 


\section{Availability of data and materials}

All data generated and analyzed during this study are included in this published article and its supplementary information files.

\section{Ethics approval and consent to participate}

This study was approved by the institutional review boards at all participating sites, and is registered with Clinicaltrials.gov (NCT00660972). All study participants provided written informed consent.

\section{Consent for publication}

Consent form available upon request.

\section{Competing interests}

NTF has served as a consultant for Gilead. MML have received grant support from Gilead. JEL has served as a consultant for Gilead and Merck, and received research support from Gilead.

\section{Author details}

'The Ohio State University, 333 W. 10th Ave. 5198 Graves Hall, Columbus, OH 43210, USA. ${ }^{2}$ University of Texas Health and Science Center, Houston, TX, USA. ${ }^{3}$ Case Western Reserve University, Cleveland, OH, USA. ${ }^{4}$ National Heart Lung and Blood Institute, Bethesda, MD, USA. 'Brigham and Women's Hospital, Harvard Medical School, Boston, MA, USA. ${ }^{6}$ Division of AIDS, National Institutes of Allergy and Infectious Diseases, National Institutes of Health $(\mathrm{NIH})$, Bethesda, MD, USA. 'University of Alabama at Birmingham, Birmingham, AL, USA.

Received: 25 September 2020 Accepted: 26 November 2020

Published online: 07 December 2020

\section{References}

1. Grunfeld CPM, Doerrler W, Shigenaga J, Jensen P, Feingold KR. Lipids, lipoproteins, triglyceride clearance, and cytokines in human immunodeficiency virus infection and the acquired immunodeficiency syndrome. J Clin Endocrinol Metab. 1992;74(5):1045-52.

2. Bowman E, Funderburg NT. Lipidome abnormalities and cardiovascular disease risk in HIV infection. Curr HIV/AIDS Rep. 2019;16(3):214-23.

3. Funderburg NT, Andrade A, Chan ES, Rosenkranz SL, Lu D, Clagett B, et al. Dynamics of immune reconstitution and activation markers in HIV+ treatment-Naiive patients treated with raltegravir, tenofovir disoproxil fumarate and emtricitabine. PLoS One. 2013;8(12):e83514.

4. Toledo E, Wang DD, Ruiz-Canela M, Clish CB, Razquin C, Zheng Y, et al. Plasma lipidomic profiles and cardiovascular events in a randomized intervention trial with the Mediterranean diet. Am J Clin Nutr. 2017;106(4): 973-83.

5. Razquin C, Toledo E, Clish CB, Ruiz-Canela M, Dennis C, Corella D, et al. Plasma lipidomic profiling and risk of type 2 diabetes in the PREDIMED trial, Diabetes Care. 2018:41(12):2617-24.

6. Shalaurova I, Connelly MA, Garvey WT, Otvos JD. Lipoprotein insulin resistance index: a lipoprotein particle-derived measure of insulin resistance. Metab Syndr Relat Disord. 2014;12(8):422-9.

7. Fernandez C, Sandin M, Sampaio JL, Almgren P, Narkiewicz K, Hoffmann M, et al. Plasma lipid composition and risk of developing cardiovascular disease. PLoS One. 2013;8(8):e71846.

8. Stegemann C, Pechlaner R, Willeit P, Langley SR, Mangino M, Mayr U, et al. Lipidomics profiling and risk of cardiovascular disease in the prospective population-based Bruneck study. Circulation. 2014;129(18):1821-31.

9. Peterson LR, Xanthakis V, Duncan MS, Gross S, Friedrich N, Volzke H, et al. Ceramide remodeling and risk of cardiovascular events and mortality. J Am Heart Assoc. 2018;7(10):e007931.

10. Belury MA, Bowman E, Gabriel J, Snyder B, Kulkarni M, Palettas M, et al. Prospective analysis of lipid composition changes with antiretroviral therapy and immune activation in persons living with HIV. Pathog Immun. 2017;2(3): 376-403.

\section{Publisher's Note}

Springer Nature remains neutral with regard to jurisdictional claims in published maps and institutional affiliations.

Ready to submit your research? Choose BMC and benefit from:

- fast, convenient online submission

- thorough peer review by experienced researchers in your field

- rapid publication on acceptance

- support for research data, including large and complex data types

- gold Open Access which fosters wider collaboration and increased citations

- maximum visibility for your research: over $100 \mathrm{M}$ website views per year

At $\mathrm{BMC}$, research is always in progress.

Learn more biomedcentral.com/submissions 